\title{
Misure spettroscopiche dirette della concentrazione dell'ozono atmosferico (*)
}

\author{
N. MITTANA
}

Ricevuto il 19 Fehbraio 1963

\begin{abstract}
Riassusto. - Questa breve relazione, presentata durante la sessione "Ottica e Spettroscopia dell'alta atmosfera" al $2^{\circ}$ Congresso Internazionale Tecnico scientifico dello spazio, concerne alcuni sistemi spettroseopici fin qui utilizzati per misure dirette dellozono atmosferico.
\end{abstract}

SUMmar. - This is a brief relation, reported during the session "Opties and Spectroscopy of the upper atmosphere" of the 2nd International Technical Scientific Meeting on Space, conceming some spectroseopical systems hitherto utilized for direct measurements of atmosperie ozone.

C'ome è noto, se tutto l'ozono rontenuto in una colonna verticale dell'atmosfera venisse isolato e portato al livello del mare, alle condizioni di temperatura e pressione normali, assumerebbe uno spessore "ridotto" di circa $3 \mathrm{~mm}$; cioè, pochi decimilionesimi dell'altezza dell'intera atmosfera omogrenea. Benché questa quantità sia tanto esigua rispetto aqui altri costituenti l'aria atmosferica, è commune sufficiente, a causa del suo forte potere assorbente sulle radiazioni luminose dell'ult ravioletto, per intereettare tutta la radiazione proenivente dal sole di lunghezza d'onda inferiore ai 2900 A, e quindi risulta sufficiente per proteggere gli esseri viventi sulla superficie terrestre da un eceesso di tali madiazioni. Senza questo schermo natumle le radiazioni ultraviolette raggiungerebbero al livello del mare una intensità superiore a cinque volte quella che si registra in alta montagna con il sole allo zenit.

$\left(^{*}\right)$ Nota presentata al 20 Congresso Internazionale Tecuico Scientifico dello Spazio. Roma, 19-23 Giugno 1962. 
Se, invece, per uno strano evento, si dovesse verificare un abnorme incremento della quantità di ozono, la vita sulla terra potrebbe diventare impossibile, come se il globo terrestre venisse avvolto da una tetra ombra biologica.

Le misure sinora effettuate delle quantità di ozono atmosferico hanno dimostrato la notevole variabilità, tanto nel tempo che nello spazio, delle concentrazioni di questo gas ed in particolare hammo messo in evidenza l'esistenza nella stratosfera, intorno ai $25-30 \mathrm{~km}$, di uno strato di massima concentrazione.

In questo strato le concentrazioni variano normalmente intorno ai valori di 2-3 parti per milione in volume d'aria, con un tenore cioè già abbastanza fastidioso per la respirazione; se si tiene conto che in qualche (aso sono state misurate concentrazioni anche di 9 p.p.m., e cioè tali da risultare probabilmente dannose per organismi superiori, e che in un prossimo futuro la stratosfera diventerà il normale ambiente dei voli umani, ne deriva immediatamente la dimostrazione dell'importanza che può assumere il problema dell'esatta valutazione delle concentrazioni atmosferiche dell'ozono anche nel campo pratico del volo stratosferico.

Già da qualche anno, molti licereatori hamo dimostrato l'esistenza di notevoli correlazioni tra le variazioni dellozono e diversi fenomeni meteorologici.

L'analisi delle fluttuazioni delle distribuzioni orizzontali e verticali dell'ozono si sta rivelando sempre più un essenziale mezzo per lo sturlio e la soluzione dei problemi relativi alla termodinamica e dinamica della stratosfera ed allo scambio energetico tra l'alta atmosfera e la troposfera, (rioè un interessante metodo di aerologia indiretta.

Il Prof. Gotz, l'insigne studioso che ha dedicato gran parte nella sua vasta attività scientifica al problema dell'ozono, ha traceciato una sintesi felice, un vivo quadro, dell'interdipendenza tra l'ozono e la fenomenologia atmosferiea:

"L'ozono ha origine, negli alti strati dell'atmosfer"a, dalla radiazione del sole, fattore primo di ogni fenomeno terrestre. A questo gas, pur tanto esiguo rispetto agli altri costituenti l'aria atmosferica, sono dovuti i contrasti termici che hamo inizio nella mesosfera, e quindi le onde ed i vortici che si producono alle quote mesosferiche dello strato caldo, al limite dell'ombla della notte polare. Il vortice polare, a sua volta, d la causa dell'abbassamento dell'ozono nella bassa stratosfera (la cosidetta "regione protetta », in quanto l'azione fotolitica sull'ozono della madiazione solare, di $\lambda<11000 \AA$, è ivi quasi trasculabile) e suceessivamente, per subsidenza e seambio turbolento, l'ozono si abbassa sino alla tropo- 
sfera, ove si realizzano i più vistosi fenomeni meteorologici; infine, diffuso sino agli strati più bassi ed in prossimità del suolo si reinserisce nel metabolismo dell'ossigeno".

Il sintetico quadro tracciato da Götz non può fornire che ma parziale visione dell'insieme dei complessi fenomeni e dei vasti problemi meteorologici ronnessi con le determinazioni delle concentrazioni dell'ozono nell'atmosfera, commque la necessità di estendere, intensificare e rendere sempre più precise le determinazioni della concentrazione dell'ozono d̀ oggi talmente sentita tra i meteorologi, che la stessa Commissione per l'Aerologia, anche recentemente, nella sua ultima riunione tenutasi l'anno scorso a Roma, ha ribadito questi concetti, formulando sull'argomento diverse raccomandazioni e definendo come "vitale " il ruolo che i dati relativi alle distribuzioni verticali dell'ozono hanno nei problemi della dinami('a stratosferica ). (Race. $7,8,9)$.

Sino a pochi anni fa l'unico metodo utilizzabile per la determinazione delle distribuzioni verticali era quello indiretto basato sull'effetto "Unkehr-Götz", che comporta una lunga serie di misurazioni spettrofotometriche, eflettuate al suolo, del rapporto delle intensita di due particolari madiazioni della banda di IIartly-Huggins, diffuse dal cielo allo zenit, durante il corso semidiurno del sole. Per applicare tale metodo necessitano osservazioni e calcolatori specializzati, e benché i calcoli siano molto laboriosi e complessi i risultati che si ottengono sono molte volte imprecisi e sempre poco dettagliati. Praticamente, con il metodo indiretto si può ottenere soltanto una valutazione qualitativa e generale della distribuzione verticale dell'ozono, ed i dati ricavabili non sono utilizzabili per mo studio sinottico delle correlazioni tra l'ozono ed i fenomeni atmosferici verificantisi a quote particolari.

Soltanto con i metodi di misura diretta, per mezzo di ozonosonde trasportate da palloni o da razzi, si possono ottenere dati sufficientemente detagliati e precisi, confrontabili con i fenomeni meteorologici a scala sinottica di specifici strati dell'atmosfera.

La prima strumentazione con la quale si son potute effettuare misure dirette delle concentrazioni dell'ozono in quota è stata quella realizzata da Regener, nel 1934, che, all'uopo, adatto un leggero spettrografo in modo da renderlo trasportabile da palloni sonda. Naturalmente, il recupero dello strumento dopo l'effettuazione del volo era la condizione necessaria per l'esame spettrofotometrico delle lastre fotografiche impiegate per la registrazione dello spettro nell'ultravioletto.

Ise stesse difficoltì comnesse al recupero, permangono per le registrazioni effettuate per mezzo di spettrografi trasportati da razzi-sonda. 
Uno dei sondaggi con razzi-sonda meglio riuscito ì stato quello realizzato dal Naval Research Jabolatory degli U.S.A. nel Giugno 1949 con il lancio di un razzo Serobee, munito di spettrografo utilizzante un reticolo di diffrazione concavo, in alluminio, di to centimetri di raggio con 15.000 tratti per pollice.

Al fine di allargare al massimo il campo di visione, in modo da poter ricevere la luce solare anche in condizioni di volo perturbato da forte rullio e deriva, è stata adoperata, invece dela normale fenditura dello spottrografo, una lente sferica di fluoruro di litio di $2 \mathrm{~mm}$ di diametro, che forniva un"immagine del sole di $13 \mu$ di diametro. Durante il volo del razzo fu effettuata una serie di esposizioni, su film particolarmente sensibile all'ultravioletto, della durata di 1 secondo, intervallate di due decimi di secondo. Contemporameamente allo spettro veniva fotografato nel film nn marcatempo.

Per determinare la quantità di ozono esistente Iungo il percorso atmosferico dei raggi solari, in corrispondenza di ciascuma esposizione fotograflea, veniva comparata la curva d'intensità di ogni speceifico spettro con le curve d'intensità deggli spettri oftenuti a quote superiori ai $100 \mathrm{~km}$, ove si ritiene che non vi è più alcuma traccia di ozono. Tenendo conto dei coefficienti di assorbimento dell'ozono per le varie Iunghezze d'onda dello spettro U.V., si potera cosi risalire alla quantità totale di ozono esistente lungo la traiettoria obliqua dei raggi solari, e conoscendo le coordinate del lazzo all'istante della registrazione fotografica, si poteva risalire al corrispondente angolo zenitale del sole e quindi alla valutazione della quantità di ozono esistente sulla verticale al disopláa del lazzo.

Alla quota di cirea $20 \mathrm{~km}$ l'assorbimento dell'ozono risultò talmente forte che poteva essere utilizzata soltanto una stretta regione dello spettro U.V., da 3200 a 3400 A. Alle quote superiori a $60 \mathrm{~km}$ era invece utilizzabile l'intero spettro a partire da $2500 \mathrm{~A}$. $\mathrm{Al}$ disopera dei $70 \mathrm{~km}$, le concentrazioni dell'ozono risultarono cosi deboli che la loro valutazione divenne troppo difficoltosa in quanto le misure rientravano neIlordine dell'errore sperimentale.

L'analisi dei dati ricavati da questo volo permise di ottenere importanti conferme di deduzioni teoriche sull'irraggiamento solare nell'ultravioletto a sull'equilibrio fotochimico dell'ozono stratosferico.

Dal rapido cenno che ho esposto si può arguire quale complessità di operazioni e quale costo comporti la realizzazione di una determinazione della distribuzione verticale dellozono per mezzo di spettrografi registratori trasportati da razzi-sonda; un tale sistema può pertanto essere utilizzato soltanto per specifiche osservazioni connesse allo studio 
di particolari importanti problemi, quali per esempio quelli dell'interdipendenza tra le fluttuazioni della radiazione ultravioletta solare, i fenomeni ionosferici e l'equilibrio fotochimico dell'ozono nell'alta atmosfera. Per la valutazione delle distribuzioni verticali dell'ozono in quota, da utilizzare nelle normali ricerche di carattere sinottico, si rende necessario pertanto l'uso di strumentazioni più exonomichè e meno complicate.

Un notevole passo avanti in questo senso fu fatto per merito di Coblenz e Stair, che nel 1939 realizzarono uno spettrofotometro oftico, che comnesso ad un moliotmasmettitore trasportato da un pallone, permetteva la ricezione a terra dei dati di misura delle intensita di due particolari lunghezze d'onda, differentemente assorbite dallozono, man mano che il pallone si elevava in quota e per mezzo dei quali potevano essere ricavati gli spessori dell'ozono attraversati dalla traiettoria obliqua dei raggi solari.

In questi ultimi anni il perfezionamento dei filtri ottici e l'uso di filtri interferenziali, atti ad isolare limitate e ben definite regioni dello spettro, ed inoltre, i recenti perfezionamenti dei sistemi radioclettrici di telemisura, specie con l'adozione di circuiti transistorizzati, hanno permesso di realizzare leggere ozonosonde ottiche trasportabili da normali palloni sonda, di sufficiente affidamento per un preciso e dettagliato computo delle concentrazioni dell'ozono alle varie quote atmosferiche.

Una delle strumentazioni di questo tipo, progettata e realizzata da Pabetzold a Kulcke, fu adoperata anche durante l'ultimo Anno Geofisico Internazionale, in un limitato numero di stazioni, con discreto suceesso.

Lozonosonda ottica di Paetzold utilizza due filtri ottici, trasparenti in una stretta regione spettrale, ampia circa 150 i, la prima centrata intorno ai 3100 i e la seconda intorno ai 3600 I. Il primo filtro è costituito dalla combinazione dei due filtri Schott U.G. 11 a GG 19 rispettivamente di 6 e $1,6 \mathrm{~mm}$ di spessore, mente il secondo filtro è composto dallo stesso U.G. 11 a da $\mathrm{un}$ WVG 1 di un mm. Quale riceritore della luce veniva adoperata una sfera di quarzo resa translucida per mezzo di una pattina di ossido di magnesio. I due filtri vengono fatti ruotare di fronte ad una cellula al selenio la cui fotocorrente di uscita risulta proporzionale allintensità delle radiazioni incidenti. Ie fotocorrenti vengono amplificate convenientemente e trasformate in radiosegnali registrabili a terra.

Poiché le radiazioni della regione spettrale centrata intorno ai 3100 \& hammo un coefficiente medio di assorbimento dell'ozono molto più alto rispetto a quelle della regione spettrale centrata intorno ai $3600 \AA$, dal rapporto delle due intensità, previa taratura, si può determinare, cono- 
scendo le coordinate geografiche e le quote del pallone sonda, lo spessore ridotto dell'ozono sovrastante l'ozonosonda.

I'ozonosonda ottica di Paetzold è stata in varie riprese sottoposta a diverse prove sperimentali e di comparazione con altre strumentazioni di tipo diverso, basate su sistemi elettrochimici di misura delle concentrazioni dell'ozono. I risultati delle prove comparative hanno confermato che le misure sono abbastanza precise e risultano sufficientemente dettagliate per la loro utilizzazione negli studi di carattere sinottico. L'elabolazione dei dati dell'ozonosonda ot tica risulta peró molto più complessa. e laboriosa rispetto a quella dell'ozonosonda elettrochimica, che tra l'altro ha il pregio di fornire direttamente le concentrazioni dell'ozono, in corrispondenza delle varie quote raggiunte durante il volo del pallone. Per ovviare alla complessitì della elaborazione dei dati dell'ozonosonda ottica, recentemente è stato progettato un sistema di valutazione dei dati stessi per mezzo di una calcolatrice elettronica.

Presso l'Osservatolio di Meteorologia Leronautica di Cagliari si stanno eseguendo delle prove sperimentali per la realizzazione di un'ozonosonda economica e di agevole uso anche da parte di personale non altamente specializzato, di tipo elettrochimico, e la sua realizzazione è già a buon punto. In un secondo tempo si cerchedì di realizzare, se i mezzi che potramno essere messi a nostra disposizione lo permetteranno, anche un'ozonosonda ottica da adoperare per scopi speciali e possibilmente razzo-trasportabile.

Si spera cosi di poter inserire, in un prossimo futuro, il Servizio Meteoroligico dell' $A$. M. italiana nel campo delle misure dirette dell'ozono atmosferico, onde fornire un sostanziale contributo anche in questo ramo della ricerea scientifica, da cui la meteorologia generale e i problemi della circolazione della stratosfera potranno trarre essenziali elementi chiarificativi. 